\title{
APENDICITE AGUDA EM GESTANTE DE 32 SEMANAS: RELATO DE CASO
}

Davi Muzi RIOS ${ }^{1}$, Vitória Conrado VIEIRA ${ }^{1}$, João Romário Gomes da SILVA ${ }^{1}$, Luciana XIMENES,2,
Gisela Machado ALTOE ${ }^{1}$, Jocieli Locatelli CERQUEIRA $^{2}$ \& Lucas Wilson Ferreira de ARAÚ JO $^{2}$

1 Universidade Iguaçu - Campus V. Itaperuna, Rio de Janeiro, Brasil.

2 Médicos residentes do Hospital São José do Avaí, serviço de Ginecologia e Obstetrícia. Itaperuna, Rio de Janeiro, Brasil.

*Autor para correspondência: davimuzirios@ hotmail.com

DOI: http://dx.doi.org/10.18571/acbm.167

\section{RESUMO}

Incidindo em 1 dentre 1500 gestações anualmente, a apendicite constitui-se em emergência cirúrgica em nosso meio, sendo a indicação mais comum de cirurgia por condições não obstétricas durante a gestação. Pode apresentar clínica inespecífica, devido às alterações anatômicas e fisiológicas advindas com a gravidez. Auxiliando na investigação diagnóstica dispomos de exames laboratoriais, e de imagem, sendo a ultrassonografia a primeira escolha. O tratamento é realizado por meio de antibióticoterapia associado a apendicectomia, podendo ser por via laparoscópica ou por laparotomia. Apresenta baixa mortalidade materna, excetuando casos de perfuração apendicular. O risco de óbito fetal eleva-se nos casos de apendicite perfurada, peritonite generalizada ou abscesso. O presente estudo tem por objetivo relatar um caso de apendicite aguda ocorrido numa gestante de 32 semanas que fora internada na maternidade do Hospital São José do Avaí, Itaperuna-RJ. Será realizada breve revisão bibliográfica em livros conceituados, além de plataformas online como Medline e Scielo, acerca do tema em pauta, observando as características gerais desta patologia e as suas peculiaridades durante o período gestacional, além de análise da conduta tomada comparando com a literatura. Concluímos que a apendicite é uma patologia relativamente comum dentre as gestantes, que embora apresente diagnóstico dificultado, este deve ser sempre suspeitado em quadros de dores abdominais e devidamente excluídos ou confirmados com a utilização de exames adequados, pois o retardo no seu diagnóstico pode elevar o risco de perfuração do apêndice, com consequente aumento do risco de óbito fetal.

Palavras chave: Gestação; Apendicite; Diagnóstico.

\begin{abstract}
Inciding in 1 out of 1500 pregnancies annually, appendicitis is a surgical emergency, being the most common indication of surgery for non-obstetric condition during pregnancy. May present non-specific clinic, due to anatomical and physiological changes due to pregnancy. Aiming at the diagnostic investigation we have laboratory tests, and imaging exams, with ultrasound being the first choice. The treatment is performed by antibiotic therapy associated with appendectomy, either laparoscopic or laparotomy. It presents low maternal mortality, except for cases of appendicular perforation. The risk of fetal death is higher in cases of perforated appendicitis, peritonitis or abscess. The present study aims to report a case of acute appendicitis occurred in a 32-week pregnant woman hospitalized in the maternity service of the Hospital São José do Avaí, ItaperunaRJ. A short bibliographical review will be carried out in well-known books, in addition to online platforms such as Medline and Scielo, about the topic in question, observing the general characteristics of this pathology and its peculiarities during the pregnancy, besides analysis of the conduct taken comparing with the literature. We conclude that appendicitis is a relatively common condition among pregnant women, that although it presents a difficult diagnosis, this must always be suspected in cases of abdominal pain and duly excluded or confirmed with the use of
\end{abstract}


appropriate exams, because the delay in its diagnosis can increase the risk of perforation of the appendix, with a consequent increase in the risk of fetal death.

Keywords: Pregnancy; Appendicitis; Diagnosis.

\section{Introdução}

Reginald Fitz em 1886 descreveu pela primeira vez a apendicite, uma patologia a qual até os dias atuais não possui etiologia completamente compreendida. Mesmo sem comprovação, considera-se a obstrução do lúmen do apêndice uma fase de importância na patogênese da apendicite. Esta obstrução, em alguns casos propicia um supercrescimento bacteriano e estiramento luminal, gerando aumento na pressão intraluminal, o que pode propiciar estase do fluxo de linfa e sangue em algumas situações, podendo ocorrer trombose vascular e necrose isquêmica, seguido de perfuração do apêndice distal (JACOBS, 2017, p. 7996-7797).

"A apendicite aguda constitui-se emergência cirúrgica em nosso meio" (FREITAS, 2009, p.38). Sua suspeição é a indicação mais comum de cirurgia por condições não obstétricas durante a gestação (FRANCA NETO, 2015, p.170). Acometendo cerca de 11 pessoas por 10000 habitantes por ano, na Europa e nas Américas, incidindo mais frequentemente na faixa etária entre 10 e 19 anos de idade, sendo o gênero masculino o mais acometido (JACOBS, 2017, p.7996). Dentre a população gestante sua incidência corresponde a 1 caso para 1500 gestações por ano, ocorrendo com maior frequência no segundo trimestre da gravidez, no período de 25 a 28 semanas, sendo mais comum em nulíparas (BOLÍVAR-RODRÍGUEZ, 2014, p.338).

Esta enfermidade é uma patologia de difícil diagnóstico, mesmo para médicos experientes, devido as várias localizações anatômicas que o apêndice pode apresentar (quadrante inferior direito até a pelve, localização típica; e flanco direito, quadrante superior direito, conforme observado em gestantes; podendo ser encontrado até mesmo no lado esquerdo do abdome nos pacientes com má rotação ou cólon severamente redundante), e também a algumas condições as quais simulam apendicite (colecistite ou outra doença da vesícula biliar, doença de Crohn, diverticulite, pneumonia de lobo inferior, doença inflamatória pélvica, gravidez ectópica, cisto ovariano roto ou outra doença cística dos ovários, etc.), devido a tais fatores "a apendicite deve ser incluída no diagnóstico diferencial de dor abdominal em qualquer faixa etária, a menos que se tenha certeza de que o órgão já foi retirado" (FREITAS, 2009, p.41; JACOBS, 2017, p. 7797).

Classicamente o quadro de apendicite aguda se inicia com anorexia sucedido de dor abdominal mal definida, por vezes um desconforto na região mesogástrica ou periumbilical, não se relacionando com atividade física ou posição, sem alívio com a eliminação de flatos ou fezes (FREITAS, 2009, p.41). Dentro de 12 a 24 horas a dor migra para o quadrante inferior direito (QID), a qual fica mais aguda, podendo ser bem localizada como inflamação transmural quando o apêndice irrita o peritônio parietal, esta irritação associa-se com a rigidez muscular local, febre até $38,3^{\circ} \mathrm{C}$ e leucocitose pode surgir no decorrer do quadro (FRANCA NETO, 2015, p.171; JACOBS, 2017, p.8001). Anorexia, náuseas e vômitos, quando presentes, ocorrem geralmente após o surgimento da dor abdominal (FRANCA NETO, 2015, p.171). No entanto nas gestantes esta apresentação clínica pode não ser muito útil, devido às alterações anatômicas e fisiológicas advindas com a gravidez, por exemplo, a localização do apêndice varia de acordo com a idade gestacional, levando a alteração no ponto doloroso (classicamente o ponto de Mc Burney), além disso, náuseas e vômitos (sintomas iniciais da apendicite) podem ser desvalorizados nesta paciente, tornando a suspeição e, consequentemente, o diagnóstico do quadro difíceis nesta etapa da vida da mulher (FREITAS, 2009, p.41; JACOBS, 2017, p.8006).

Mas apesar da apresentação clássica ser menos provável de ocorrer nas gestantes, os sintomas mais comuns da apendicite, a dor no QID ocorre perto ao ponto de McBurney na maioria das gestações, independente da idade gestacional do feto (FRANCA NETO, 2015, p.171). 
Dentre os achados laboratoriais temos leucograma variando entre 10000 a 18000 leucócitos $/ \mathrm{mm}^{3}$, tendo média de 15000 leucócitos $/ \mathrm{mm}^{3}$ com desvio para a esquerda, no entanto a leucocitose pode ser um achado normal durante a gestação, variando de 16900 leucócitos $/ \mathrm{mm}^{3}$ no terceiro trimestre até valores de 29000 leucócitos $/ \mathrm{mm}^{3}$ durante o trabalho de parto, inclusive a presença de desvio para a esquerda (FRANCA NETO, 2015, p.171; FREITAS, 2009, p.43).

O diagnóstico desta condição parte de uma boa anamnese, atentando-se para as queixas e a condição clínica da paciente, e aos exames solicitados, mesmo que os achados sejam confusos, a apendicite, como citado por Jacobs (2017) deve ser um diagnóstico diferencial em casos de dor abdominal, excetuando as pacientes sabidamente apendicectomizadas. Aliado à suspeição clínica dispomos de diversos exames de imagem, tais como: radiografia; ultrassonografia (USG); tomografia computadorizada (TC); ressonância magnética (RNM) (FREITAS, 2009, p.45-46).

Na gestação a investigação com o auxilio da imagenologia inicia-se com a ultrassonografia, a qual, mesmo que o apêndice aparente ser normal o diagnóstico não deve ser excluído, exceto nos casos em que a USG encontre achados sugestivos de outras patologias (como a torção ovariana e nefrolitíase), Caso esta seja inconclusiva, pode-se lançar mão da RNM, a qual apresenta bons resultados e também é uma boa alternativa no intuito de evitar exposição à radiação ionizante nos casos de radiografia e TC, sendo esta ultima indicada nos casos em que os achados clínicos e a USG forem inconclusivos ou a RNM não estiver disponível para uso (FRANCA NETO, 2015, p.173).

O tratamento desta afecção deve incluir antibioticoterapia, que deve abranger bactérias Gram-negativas, Gram-positivas e anaeróbios, além da realização de cirurgia, podendo ser por via laparoscópica ou por laparotomia, de acordo com a experiência do cirurgião, sendo a via laparoscópica bastante vantajosa para a paciente, no entanto necessita de avaliação criteriosa quanto a possíveis riscos para o feto (FRANCA NETO, 2015, p.174; BOLÍVAR-RODRÍGUEZ, 2014, p.338; DALAQUA, CORSI, 2006, p.8).

A mortalidade materna após apendicectomia é semelhante às pacientes não grávidas, exceto nos casos de perfuração do apêndice (FRANCA NETO, 2015, p.174). Franca Neto diz que o risco de óbito fetal se eleva nos casos de apendicite perfurada, peritonite generalizada ou abscesso, Bolívar-Rodríguez (2014) corrobora ao citar que nos casos de apendicite não perfurada a mortalidade fetal atinge 5\% enquanto que nos casos de perfuração este valor sobe para 36\%, sendo que a ruptura apendicular ocorre mais frequentemente no terceiro trimestre da gestação.

O presente estudo tem por objetivo relatar um caso de apendicite aguda ocorrido numa gestante de 32 semanas que fora internada na maternidade do Hospital São José do Avaí, Itaperuna-RJ.

\section{Relato de caso}

T.V.S., 22anos, G2PC1A0, 31s+6d. Deu entrada na maternidade do Hospital São José do Avaí no dia 09/10/17 com dor abdominal difusa tipo contração que iniciou ha 1 dia. Tabagista, em uso de 10 cigarro ao dia.

Ao exame: bom estado geral, hidratada, corada, eupneica, afebril, normotensa pressão arterial 100x60 mmhg, frequência cardíaca $88 \mathrm{bpm}$, temperatura de $37^{\circ} \mathrm{C}$. Abdome gravídico, fundo de uterino $28 \mathrm{~cm}$, distendido, hipertimpanico, doloroso a palpação superficial e profunda, sem sinais de irritação peritoneal. Evoluiu pela manhã com dispnéia, taquicardia e sinais de irritação peritoneal. Foi realizado exames de imagem (USG e Tomografia Computadorizada de abdômen) e exames laboratoriais que evidenciaram feto único, situação longitudinal, apresentação cefálica e dorso a esquerda, frequência cardíaca fetal de 160 bpm, movimento fetal diminuído, adramnia e líquido livre em cavidade abdominal. Leucograma 15.600 com desvio para esquerda.

Paciente foi submetida a cesariana de emergência com auxílio da equipe de cirurgia geral. Cesariana sem intercorrências, porém, durante revisão da cavidade abdominal foi visualizado 
fecalito e apendice perfurado. Realizada apendicectomia, inserido dreno de cavidade. A paciente foi encaminhada ao CTI geral do mesmo nosocômio e iniciado esquema tríplice de antibioticoterapia (Amicacina, Metronidazol e Ampicilina). Esteve 5 dias sob cuidados intensivos e encaminhada para enfermaria onde permaneceu mais 2 dias para completar esquema de antibiótico.

Recém-nascido encaminhado a UTI Neonatal onde teve boa evolução recebendo alta após 18 dias de internação.

\section{Discussão}

$\mathrm{Na}$ internação a paciente queixava-se de dor abdominal difusa de início há 01 dia, encontrava-se afebril, sem demais queixas, sendo prescrito sintomáticos, os quais aliviaram parcialmente os sintomas. Ela vinha de tratamento para pielonefrite, sendo esta afecção o diagnóstico diferencial de apendicite mais comum na gravidez (FIGUEIREDO e CORSO, 2002, p.285). Apesar do quadro álgico por ela apresentado também foi chamado a atenção para a divergência observado entre a medida do fundo de útero, com $28 \mathrm{~cm}$, e a idade gestacional, de 32 semanas, valores que deveriam corresponder, pois segundo elucida Montenegro e Rezende Filho (2013) a partir de 20 semanas de gestação, quando o útero se encontra a nível da cicatriz umbilical até a 32 semana, o valor obtido na medida do fundo de útero corresponde à idade gestacional do feto. Achado este que levantou a hipótese de crescimento intrauterino restrito (CIUR), que de acordo com Montenegro e Rezende Filho (2013, p514) "define o feto que não consegue atingir o seu potencial genético de crescimento", apresentando diversas etiologias, podendo ser fetal, placentário ou materno, sendo a principal causa a insuficiência placentária (Montenegro e Rezende Filho, 2013, p.514), possuindo no tabagismo um fator de risco para esta, pois como explica Kulay Junior et al. (2013) a nicotina possui efeito vasoconstritor arteriolar podendo causar redução na perfusão placentária. Visto isso foi solicitado o exame de cardiotocografia fetal, o qual obteve-se resultado dento dos parâmetros da normalidade, sendo o feto reativo mediante estímulo sonoro.

Pela manhã a paciente queixa-se de intensa dor abdominal, com sinais de irritação peritoneal mais sensível em região periumbilical associado a dispneia. O que levantou a suspeita de uma possível apendicite em curso, sendo solicitado ultrassonografia abdominal total de urgência e ultrassom obstétrico.

Dentre os exames laboratoriais observou-se leucocitose (14600 leucócitos/ml) com presença de desvio para a esquerda ( $16 \%$ de bastonetes), o que mesmo podendo ser um estado fisiológico proporcionado pela gestação, aliado à clínica da paciente reforçou a suspeita inicial (FRANCA NETO, 2015, p.171; FREITAS, 2009, p.43).

A USG obstétrica confirmou os achados do primeiro exame físico ao evidenciar anidramnia, reduzida movimentação fetal, compatível com CIUR, possivelmente devido ao tabagismo. (KULAY JUNIOR et al. 2013, p883).

A USG abdominal total foi inconclusiva, o que levantou a necessidade de lançar mão da tomografia computadorizada para elucidar o diagnóstico. A utilização de tal método diagnóstico não é totalmente contraindicada, dado as necessidades específicas do caso em pauta, envolvendo risco de vida para a gestante e seu concepto. Tendo em vista a segurança do embrião D'Ippolito e Medeiros (2005) elucidam que o período de maior sensibilidade aos efeitos da exposição a radiação ionizante corresponde às duas primeiras semanas de gestação, em que o embrião poderá permanecer intacto, ser reabsorvido, ou abortado. No entanto após a $32^{\mathrm{a}}$ semana de gestação estes autores dizem que, salvo um aumento possível no risco de desenvolvimento de uma neoplasia maligna no decorrer da infância ou de sua maturidade, não haverá riscos graves ao feto, portanto dado ao maior benefício em função do risco em potencial, optou-se por executar a tomografia computadorizada sem contraste (Figuras 1A e 1B). 


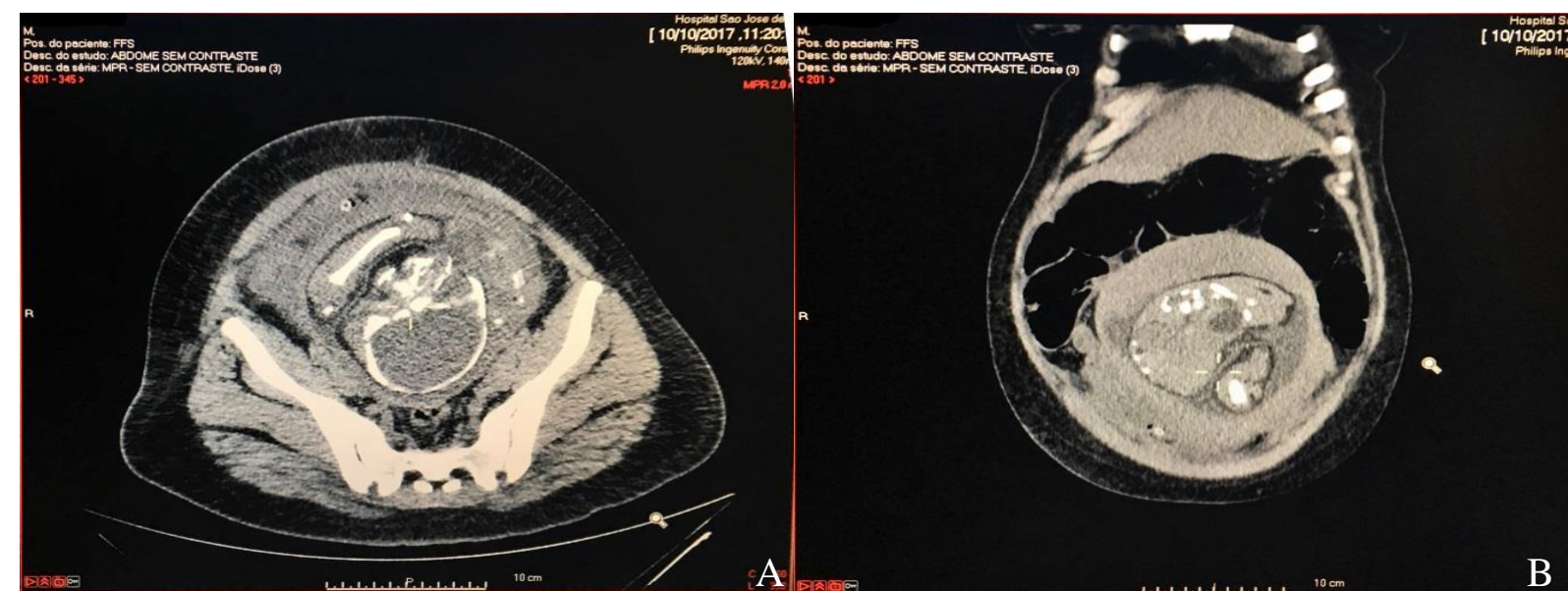

Figura 1: (A) tomografia de abdome em corte axial evidenciando cavidade uterina preenchida por feto único, além de imagem hiperdensa, arredondada, com presença de gás em sua adjacência, em hemiabdome direito.

(B) Tomografia de abdome em corte coronal, demonstrando presença de feto, único, dentro de cavidade uterina, reduzida quantidade de líquido amniótico e, pequena imagem hiperdensa, arredondada, associado a imagem hipodensa adjacente (gás) em hemiabdome direito.

A figura 1A observamos feto único, em posição cefálica, dorso a esquerda, reduzida quantidade de líquido amniótico. Sendo que nas figuras $1 \mathrm{~A}$ e $1 \mathrm{~B}$ é possível notar imagem hiperdensa, circular, bem definida, com presença de gás em sua adjacência em hemiabdome direito. No entanto tal exame apresentou resultado inconclusivo quanto a suspeita diagnóstica.

Dado ao quadro apresentado pela paciente optou-se por submete-la a cesariana de emergência com o auxílio da equipe de cirurgia geral. A cesariana ocorreu sem intercorrências, no entanto, durante revisão da cavidade abdominal foi observado fecalito e apêndice perfurado, sendo realizada respectiva apendicectomia e inserido dreno de cavidade.

Posterior a cirurgia a paciente foi encaminhada ao CTI geral do nosocômio e iniciado esquema antibiótico tríplice (Amicacina, Metronidazol e Ampicilina), permanecendo por 5 dias, sendo posteriormente transferida para a enfermaria para completar o esquema antibiótico.

O recém-nascido foi encaminhado a UTI Neonatal onde teve boa evolução recebendo alta após 18 dias de internação.

\section{Conclusão}

Concluímos que a apendicite é uma patologia relativamente comum dentre as gestantes, que embora apresente diagnóstico dificultado devido as alterações fisiológicas que ocorrem no organismo materno, este deve ser sempre suspeitado em quadros de dores abdominais e devidamente excluídos ou confirmados com a utilização de exames adequados, pois o retardo no seu diagnóstico pode elevar o risco de perfuração do apêndice, com consequente aumento do risco de óbito fetal.

\section{Bibliografia}

BOLÍVAR-RODRÍGUEZ, MA; et al. Acute appendicitis during pregnancy: report of 4 cases. Ginecologia Y Obstetricia De Mexico. Mexico, 82, 5, 337-343, May 2014. ISSN: 0300-9041.

DALAQUA, Mariana; CORSI, Paulo Roberto. Apendicite aguda na gestação. Arq Med Hosp Fac Cienc Med Santa Casa São Paulo 2006; 51(1):4-9. Disponível em: < http://www.fcmsantacasasp.edu.br/images/Arquivos_medicos/2006/51_1/vlm51n1_1.pdf>. Acessado em 26 de outubro de 2017. 
D'IPPOLITO, Giuseppe; MEDEIROS, Regina Bitelli. Exames radiológicos na gestação. Radiol Bras, São Paulo, v. 38, n. 6, p. 447-450, Dec. 2005. Disponível em: $<$ http://www.scielo.br/scielo.php?script=sci_arttext\&pid=S0100-

39842005000600013\&lng=en\&nrm=iso>. Acessado em 30 de outubro de 2017. http://dx.doi.org/10.1590/S0100-39842005000600013.

FIGUEIREDO, Fernando Antônio Santos; CORSO, Carlos Otávio. Apendicectomia laparoscópica na gestante. Rev. Col. Bras. Cir., Rio de Janeiro, v. 29, n. 5, p. 284-287, Oct. 2002. Disponível em: $\quad<$ http://www.scielo.br/scielo.php?script=sci_arttext\&pid=S010069912002000500007\&lng=en\&nrm=iso>. Acessado em 30 de outubro de 2017. http://dx.doi.org/10.1590/S0100-69912002000500007.

FRANCA NETO, Antônio Henriques de; AMORIM, Melania Maria Ramos do and NOBREGA, Bianca Maria Souza Virgolino. Apendicite aguda na gestação: revisão de literatura. Rev. Assoc. Med. Bras. [online]. 2015, vol.61, n.2, pp.170-177. ISSN 0104-4230.

FREITAS, RG; PITOMBO, MB; MAYA, MCA; LEAL, PRF. Apendicite aguda. Revista Hospital Universitário Pedro Ernesto. 2009;8(1):38-51. Disponível em: <http://revista.hupe.uerj.br/detalhe_artigo.asp?id=168\#citar>. Acessado em 24 de outubro de 2017.

KULAY JUNIOR, Luiz et al. Efeitos iatrogênicos de medicamentos e de imunizações. Farmacodependência. Infortunística. In: Rezende Obstetrícia. 12.ed. Rio de Janeiro: Guanabara Koogan, 2013. p.883

MONTENEGRO, Carlos Antonio Barbosa; REZENDE FILHO, Jorge de. Idade da gestação e data provável do parto. In: Rezende Obstetrícia. 12.ed. Rio de Janeiro: Guanabara Koogan, 2013. p.167.

Crescimento intrauterino restrito. In: Rezende Obstetrícia. 12.ed. Rio de Janeiro: Guanabara Koogan, 2013. p.514..

JACOBS, Danny O. Apendicite aguda e peritonite. In: Medicina interna de Harrison [recurso eletrônico] / [Dennis L.] Kasper ... [et al.]; tradução: Ademar Valadares Fonseca ... et al. [revisão técnica: Alessandro Finkelsztejn ... et al]. - 19. ed. - Porto Alegre: AMGH, 2017. e-PUB. 